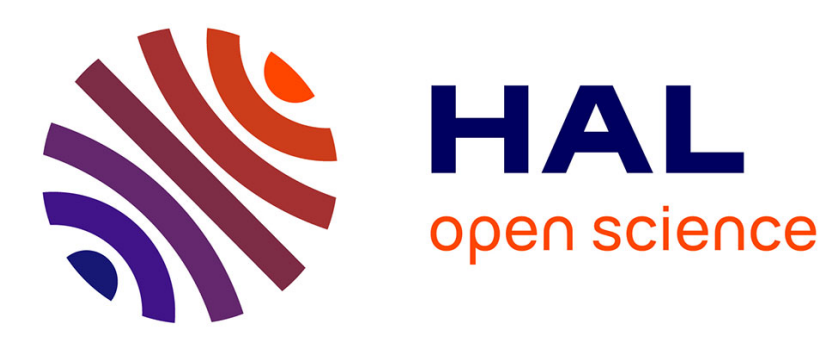

\title{
A DNP-hyperpolarized solid-state water NMR MASER: observation and qualitative analysis
}

Emmanuelle Weber, Dennis Kurzbach, Daniel Abergel

\section{To cite this version:}

Emmanuelle Weber, Dennis Kurzbach, Daniel Abergel. A DNP-hyperpolarized solid-state water NMR MASER: observation and qualitative analysis. Physical Chemistry Chemical Physics, 2019, 21 (38), pp.21278-21286. 10.1039/C9CP03334C . hal-02337358

\section{HAL Id: hal-02337358 \\ https://hal.sorbonne-universite.fr/hal-02337358}

Submitted on 29 Oct 2019

HAL is a multi-disciplinary open access archive for the deposit and dissemination of scientific research documents, whether they are published or not. The documents may come from teaching and research institutions in France or abroad, or from public or private research centers.
L'archive ouverte pluridisciplinaire HAL, est destinée au dépôt et à la diffusion de documents scientifiques de niveau recherche, publiés ou non, émanant des établissements d'enseignement et de recherche français ou étrangers, des laboratoires publics ou privés. 


\title{
A DNP-hyperpolarized solid-state water NMR
}

\section{MASER: observation and qualitative analysis}

\author{
Emmanuelle Weber, ${ }^{\dagger}$ Dennis Kurzbach, ${ }^{\dagger, \dagger}$ and Daniel Abergel ${ }^{*, \dagger}$ \\ Laboratoire des biomolécules, LBM, Département de chimie, Ecole normale supérieure, PSL \\ University, Sorbonne Université, CNRS, 75005 Paris, France. \\ E-mail: daniel.abergel@ens.fr
}

\begin{abstract}
We present observations of an NMR maser (microwave amplification by stimulated emission of radiation) of hyperpolarized ${ }^{1} \mathrm{H}$ nuclei by dynamic nuclear polarization (DNP) at $1.2 \mathrm{~K}$ and in a magnetic field of $6.7 \mathrm{~T}$. The sustained maser pulses originate from the interplay between radiation damping (RD) due to the large ${ }^{1} \mathrm{H}$ magnetization, and the remagnetization to a negative value by the DNP process. NMR signals lasting for several tens of seconds are thus observed on an ensemble of dipolar-coupled nuclear spins. Magnetization dynamics are analyzed in terms of the combined Bloch-Maxwell and Provotorov (BMP) equations for RD and DNP. Insight into the long time evolution of the magnetization is provided by a theoretical analysis of this nonlinear dynamical system, and by fitting the NMR signal to a simplified version of the BMP equations.
\end{abstract}

A large magnetization can exhibit unconventional behaviour that departs from the dynamics in a static magnetic field predicted by the simple Bloch equations, in cases of

\footnotetext{
*To whom correspondence should be addressed

†Laboratoire des biomolécules, LBM, Département de chimie, Ecole normale supérieure, PSL University, Sorbonne Université, CNRS, 75005 Paris, France.

${ }^{\ddagger}$ Present address: Univerität Wien, Fakultät für Chemie, Institut für biologische Chemie, Währinger Strasse 38, 1090 Wien, Österreich
} 
strong coupling with the detection circuit. This phenomenon, designated as "radiation damping" (RD), was already observed in the early days of nuclear magnetic resonance (NMR). ${ }^{1}$ Radiation damping can be described in classical terms through the so-called Bloch-Maxwell equations that account for the radio-frequency (rf) field that is induced in the coil of the detection circuit through precession of the magnetization. ${ }^{1,2}$ RD thus induces collective and coherent motions of the spins that are slaved to each other upon the action of a feedback field from the detection circuit, a process that is analogous to superradiance. ${ }^{3}$ Thus, RD can be described as an NMR MASER. This is an active process, in contrast to other types of coherent processes, such as spin echoes, that occur passively upon the spin manipulation by an externally applied RF field. In liquid-state NMR, RD is typically observed when a large solvent magnetization, usually water, is present. Radiation damping may give rise, in some instances, to unexpected features, such as typical nonlinear maser pulses, multiple echoes, ${ }^{4}$ or the appearance of spurious harmonics in two-dimensional liquid state $\mathrm{NMR}^{2,5-7}$ which are related to the nonlinear nature of the modified Bloch-Maxwell equations. In certain circumstances, when magnetization is continuously replenished, this gives rise to characteristic multiple spontaneous signal bursts, leading to sustained maser oscillations. Such manifestations have been previously observed for hyperpolarized spin states that exceed typical magnetization levels by several orders of magnitude, for optically pumped noble gases, ${ }^{8}$ in the case of dissolved hyperpolarized ${ }^{129}$ Xe when distant dipolar field effects are effective, ${ }^{9}$ or on thermally polarized water at room temperature through electronic control of the coil rf feedback field. ${ }^{10,11}$ Alternatively, similar unconventional magnetization dynamics were observed in the context of dynamic nuclear polarization (DNP) for polarized targets, ${ }^{12,13}$ or in the case of a ruby crystal.

Here, we investigate such NMR maser effects in the framework of high-field DNP. This is motivated by the recent developments in DNP methodology that clearly tend towards ever higher polarization levels and larger magnetizations. In this context, intense radiation damping may well interfere with rf pulse sequences used in practice, such as cross-polarization, to reduce their efficiencies. We present observations of a ${ }^{1} \mathrm{H}$ 
NMR maser on a DNP-hyperpolarized water-glycerol sample at $1.2 \mathrm{~K}$, in a $6.7 \mathrm{~T}$ magnet, where the recorded induction signal could be observed for more than a second, and up to several tens of seconds in some cases, even after microwave irradiation has been switched off. Following previous studies, ${ }^{14}$ these observations of this high-field DNP maser were rationalized in terms of the Maxwell-Bloch-Provotorov equations. This shows that the collective behaviour of an ensemble of dipolar-coupled nuclear spins can be taken into account by a simple vector model.

Hyperpolarization of nuclei through DNP is achieved by transferring part of the high electron spin polarization (nearly $100 \%$ at temperatures approaching $1 \mathrm{~K}$ ) of paramagnetic impurities to nearby nuclear spins via hyperfine and superhyperfine couplings. Depending on the experimental conditions, various mechanisms can contribute more or less efficiently to DNP. Importantly, these depend on the electron paramagnetic resonance (EPR) line characterizing the paramagnetic impurities. A great amount of work has been devoted to the elucidation of these complex aspects since the early days of DNP (see for instance previous work ${ }^{16-19}$ ), and this field of research has regained interest in a more recent period with the renewal of DNP techniques, such as dissolution-DNP ${ }^{20}$ and the introduction of DNP in MAS solid-state NMR. ${ }^{21}$

In the study at hand, the EPR linewidth of the paramagnetic impurity is significantly larger than the NMR transition frequency, so that the hyperpolarization process of the nuclei mainly involves a three-spin process, i.e. a flip-flop between an electron spin and a nuclear spin associated to a flip or a flop of one additional electron spin. ${ }^{22,23}$ Moreover, in the "thermodynamic limit", where large ensembles of spins are involved in the process, DNP can be described in terms of thermal mixing (TM), where the density matrix is defined by an instantaneous spin temperature of several, possibly coupled, heat reservoirs. ${ }^{16,24-26}$ In other words, in the TM limit, the DNP process can be described by a Zeeman nuclear spin heat reservoir and an electron dipole-dipole reservoir, the temperatures of which eventually tend to equilibrate to a common spin temperature. Provotorov ${ }^{24,25}$ derived kinetic equations, valid in the high-temperature limit, that characterize the time-dependence of the inverse spin temperatures of the 
electron spin Zeeman and dipolar reservoirs, and of the nuclear Zeeman reservoir. According to his theory, the nuclear spin temperature $\beta_{n}(t)$ equilibrates with $\beta_{e e}(t)$, the spin temperature of the dipole-dipole electron spin reservoir. This model can be extended to the case where several nuclear spin species are present in the sample, each acting as a reservoir and assigned a spin temperature.

The high nuclear polarization levels achieved by DNP have dramatic consequences on the dynamics of the magnetization. Indeed, the coupling with the high-Q detection circuit of the NMR probe yields a significant radio-frequency feedback field from the detecting coil, thereby leading to efficient radiation damping, analyzed in terms of the Bloch-Maxwell equations. ${ }^{1,27}$ When the probe of the NMR spectrometer is tuned to the frequency of the spins, the dynamics of the magnetization $M$ in a frame rotating around the $z$ axis at the frequency $\omega$ obeys a set of modified Bloch equations.

Combining the theories of TM-DNP and RD, Bösiger et al. proposed the following set of dynamical equations to describe the nuclear magnetization and the various inverse spin temperatures involved: ${ }^{1}$

$$
\left\{\begin{array}{l}
\dot{M}_{x}=\delta \omega_{H} M_{y}-\gamma_{\mathrm{H}} G M_{z} M_{x}-\frac{M_{x}-M_{x}^{t h}}{T_{2}} \\
\dot{M}_{y}=-\delta \omega_{H} M_{x}-\omega_{1} M_{z}-\gamma_{\mathrm{H}} G M_{z} M_{y}-\frac{M_{y}-M_{y}^{t h}}{T_{2}} \\
\dot{M}_{z}=\omega_{1} M_{y}+\gamma_{\mathrm{H}} G\left(M_{x}^{2}+M_{y}^{2}\right)-\frac{\gamma_{\mathrm{H}}^{2} \hbar^{2} \mathcal{N}_{\mathrm{H}} B_{0}}{4 k_{\mathrm{B}}} \gamma_{n, e e}\left(\beta_{n}-\beta_{e e}\right) \\
\frac{d \beta_{e e}}{d t}=-\gamma_{e e, n}\left(\beta_{e e}-\beta_{n}\right)-\gamma_{e e, d}\left(\beta_{e e}-\beta_{d}\right)-\frac{1}{\tau_{e e, L}}\left(\beta_{e e}-\beta_{L}\right) \\
+\pi \omega_{1 \mu w}^{2} g\left(\Delta \omega_{e}\right) \frac{\Delta \omega_{e}}{\delta \omega^{2}}\left[\omega_{0 e} \beta_{e}-\Delta \omega_{e} \beta_{e e}\right] \\
\frac{d \beta_{e}}{d t}=\gamma_{e e, \mathrm{~L}}\left(\beta_{e}-\beta_{L}\right)+\pi \omega_{1}^{2} g\left(\Delta \omega_{e}\right)\left(\frac{\Delta \omega_{e}}{\omega_{0 e}} \beta_{e e}-\beta_{e}\right) \\
\frac{d \beta_{d}}{d t}=-\gamma_{d, e e}\left(\beta_{d}-\beta_{e e}\right)
\end{array}\right.
$$

In these equations, the first three describe the magnetization dynamics due to RD, and $G=\frac{\mu_{0}}{2} \eta Q$, where $\eta$ and $Q$ are respectively the filling factor and the quality factor of the probe's detection circuit, $\mu_{0}$ is the vacuum permeability. The rf field $\omega_{1}$ (in frequency units) is aligned along $x$ in the rotating frame, and $\delta \omega_{H}=\omega-\omega_{0}$ is the frequency offset with respect to the Larmor frequency $\omega_{0}$ of the spins. The last three equations 
of Eq. 1 are the Provotorov equations, ${ }^{25}$ where $\omega_{0 e}$ is the electron resonance frequency, $g(\omega)$ and $\delta \omega_{e}$ are the lineshape and linewidth of the EPR line, $\omega_{1 \mu w}$ and $\omega_{\mu w}$ are the magnitude and frequency of the microwave irradiation field, and $\Delta \omega_{e}=\omega_{0 e}-\omega_{\mu w}$. The nuclear and electron dipolar inverse spin temperatures are $\beta_{n}$ and $\beta_{e e}$, and the lattice temperature is $\beta_{\mathrm{L}} \cdot \gamma_{n, e e}=\tau_{n, e e}^{-1}$ is the kinetic rate of inverse spin temperature transfer from the nuclear Zeeman to the electronic dipolar spin reservoir, and $\gamma_{e e, n}=$ $\frac{c_{n}}{c_{e}} \gamma_{n, e e}$, where the coefficients $c_{n}$ and $c_{e}$ denote the heat capacities of the associated reservoirs. It should be noted that in Eqs 1 the spin temperature $\beta_{d}$ of the deuterium spin reservoir is coupled to the electron dipolar reservoir with the rate constant $\gamma_{d, e e}$, so that the former becomes indirectly coupled to the ${ }^{1} \mathrm{H}$ reservoir. Therefore, polarization transfer between both nuclear species may occur indirectly, through coupling with the electron dipolar reservoir. Also, the expression of $M_{z}$ in Eq. 1 has been replaced by $M_{z}=\frac{\gamma_{\mathrm{H}}^{2} \hbar^{2} \mathcal{N}_{\mathrm{H}} B_{0}}{4 k_{\mathrm{B}}} \beta_{n}$, in order to emphasize the link between the Bloch equations and the thermal mixing description. The theoretical set-up described by Eqs. 1 is illustrated in Fig.1.

The nuclear spin-lattice relaxation time is usually much longer than the other characteristic times relevant to the problem ( $\sim$ minutes), and can therefore be neglected. Rather, the z-component of the magnetization returns to a state of common temperature with the electron dipole-dipole reservoir, with the time constant $\gamma_{n, e e}$. Finally, it has been postulated ${ }^{14}$ that in Eqs. 1 the transverse components of the magnetization relax towards non-zero values $M_{x}^{t h}$ and $M_{y}^{t h}$ caused by the presence of a Nyquist noise voltage in the detection circuit and the resulting excitation of the magnetization.

For the study at hand, experiments were performed at $1.2 \mathrm{~K}$ in a Bruker prototype polarizer operating at a $6.7 \mathrm{~T}$ magnetic field $\left(285 \mathrm{MHz}{ }^{1} \mathrm{H}\right.$ Larmor frequency). A continuous microwave irradiation at $188.380 \mathrm{GHz}$ was provided by a a $94 \mathrm{GHz}$ source (ELVA-1) coupled to a frequency doubler (Virginia Diodes). The nominal output power of the source was $350 \mathrm{~mW}$, but the power reaching the sample was estimated to be on the order of $\sim 12 \mathrm{~mW}$. DNP experiments were performed on a $150 \mu \mathrm{L}$ sample composed of $10 \%$ of $\mathrm{H}_{2} \mathrm{O}, 40 \%$ of $\mathrm{D}_{2} \mathrm{O}$ and $50 \%$ of deuterated glycerol $\left(\mathrm{C}_{3} \mathrm{D}_{8} \mathrm{O}_{3}\right)$ as a glass-forming 
agent to avoid crystallization upon freezing in liquid He. The sample was contained in a cylindrical PTFE cup in order to avoid spurious ${ }^{1} \mathrm{H}$ signals. TEMPOL (4-hydroxy2,2,6,6-tetramethylpiperidine-1-oxyl) was used as paramagnetic agent, at a final concentration of $50 \mathrm{mM}$, as it is known to efficiently polarize nuclear spins with large gyromagnetic ratios, such as ${ }^{1} \mathrm{H}$. The $\mu$-wave frequency was chosen so as to achieve a negative nuclear polarization. Typical ${ }^{1} \mathrm{H}$ NMR linewidths were c.a. $25 \mathrm{kHz}$, due to dipolar interactions of the nuclear spins. When the microwave irradiation frequency is chosen so as to polarize nuclei negatively (that is, to create a ${ }^{1} \mathrm{H}$ magnetization pointing towards $-z$ ), the FID envelope changes dramatically as soon as the magnetization reaches some threshold value. Then, FIDs exhibit a series of signal revivals extending over a few tens of ms, evocative of maser bursts, with intensities that gradually decrease with time. At times longer than $\sim 100 \mathrm{~ms}$, the FIDs keeps decaying very slowly, allowing signal persistence for times up to several tens of seconds. This behaviour is highly evocative of the presence of strong RD. This is confirmed by the disappearance of these magnetization revivals when the receiver circuit is detuned, thereby reducing the magnetization-coil coupling. Similar behaviours were observed once the samples have been polarized, irrespective of the persistence or not of $\mu$-wave irradiation during acquisition. Typical observations are shown in Fig. 2.

These sustained maser pulses result from the competition between two physically different processes: i) a large magnetization strongly coupled to the NMR detection circuit, responsible for a radio frequency feedback field from the probe that drives the magnetization towards its equilibrium position, aligned with the magnetic induction field; and ii) the growth in magnitude of the $M_{z}$ component of the magnetization, towards a state of negative polarization through DNP.

Because of the non-linearity of the BMP equations, the dynamics of the magnetization are difficult to grasp. Moreover, as is often the case for nonlinear differential systems, even finding explicit stationary solutions is already challenging. Thus, no general rules or criteria allow one to predict the time evolution, and only extensive numerical studies can provide a thorough account of the dynamics contained within the 
model. Nevertheless, qualitative information may still be obtained on the system at hand. Here, we show what insight can be gained by analysis of the evolution at short times. Moreover, owing to some simplifications that will be detailed below, explicit expressions of the stationary solutions - the fixed points of the differential system - can be found. This allows for the investigation of the long-time magnetization dynamics through the study of the behaviour of the linearized differential system in the vicinity of the fixed points. ${ }^{29}$

In the case of the complete BMP problem (Equation 1), even the determination of the stationary solutions is challenging, and so is the determination of the evolution of the magnetization and of the nuclear and electron dipole spin temperatures near the fixed points. Therefore, for qualitative analyses, simplifying assumptions were used: Firstly, the electron Zeeman spin temperature $\beta_{e}$ was assumed constant and equal to the lattice temperature $\beta_{L}$ (an assumption used in ${ }^{14}$ ), and secondly, the small circuit noise term is neglected, so that the constants $M_{x, y}^{t h} / T_{2}$ are set to zero. Taking into account these simplifications, Eqs. 1 can be rewritten with the use of the new variables $u(t)=M_{x}^{2}+M_{y}^{2}$, with $M_{x}(t)+i M_{y}(t)=\sqrt{u} e^{i \phi(t)}$; and $w(t)=\frac{\gamma_{\mathrm{H}}^{2} \hbar^{2} \mathcal{N}_{\mathrm{H}} B_{0}}{4 k_{B}} \beta_{e e}=B_{h} \beta_{e e}$, and $w_{\mathrm{L}}^{0}=B_{h} \beta_{\mathrm{L}}$. Here, $w_{\mathrm{L}}^{0}$ is just the ${ }^{1} \mathrm{H}$ nuclear thermal magnetization. With these new variables, and introducing the notations $M_{z}(t) \rightarrow z(t), T 2 \rightarrow \gamma_{2}^{-1}$ and $\gamma G \rightarrow \lambda$, Eqs. 1 become:

$$
\begin{aligned}
\dot{u}(t) & =-2\left(\lambda z(t)+\gamma_{2}\right) u(t) \\
\dot{z}(t) & =\lambda u(t)-\gamma_{n, e e}(z(t)-w(t)) \\
\dot{\phi}(t) & =-\delta \omega_{H} \\
\dot{w}(t) & =-\gamma_{e e, n}(w(t)-z(t))-\gamma_{e e, d} w(t)+\gamma_{e e, d} B_{h} \beta_{d}(t)-\gamma_{e e, \mathrm{~L}}\left(w(t)-w_{\mathrm{L}}^{0}\right)-\delta w(t)+\Omega w_{\mathrm{L}}^{0} \\
\dot{\beta}_{d}(t) & =-\gamma_{d, e e}\left(\beta_{d}(t)-\frac{1}{B_{h}} w(t)\right)
\end{aligned}
$$

, where the rates $\gamma_{e e, n}$ and $\gamma_{e e, d}$ are: $\gamma_{e e, n}=\frac{c_{\mathrm{H}}}{c_{\mathrm{E}}} \gamma_{n, e e}$ and $\gamma_{e e, d}=\frac{c_{\mathrm{D}}}{c_{\mathrm{E}}} \gamma_{d, e e}$. The equation for $\dot{w}(t)$ can be rewritten as: $\dot{w}(t)=b z(t)+a w(t)+d \beta_{d}(t)+c$, where $a=-\gamma_{e e, n}-\gamma_{e e, \mathrm{~L}}-\gamma_{e e, d}-\delta$, $b=\gamma_{e e, n}, c=\left(\gamma_{e e, \mathrm{~L}}+\Omega\right) w_{\mathrm{L}}^{0}, d=\gamma_{e e, d} B_{h}$. In these equations, $\delta$ and $\Omega$ are defined as: $\Omega=\pi \omega_{1 \mu w}^{2} g\left(\Delta \omega_{e}\right) \frac{\Delta \omega_{e}}{\delta \omega^{2}} \omega_{0 e}$ and $\delta=-\pi \omega_{1 \mu w}^{2} g\left(\Delta \omega_{e}\right) \frac{\Delta \omega_{e}^{2}}{\delta \omega^{2}}$. where $\Delta \omega_{e}$ is defined above. 
Some insight into the sustained maser period of the FIDs can be obtained based on qualitative arguments and numerical simulations of Eqs. 2, and are listed below:

- If the initial time of each signal burst is defined as the time when the FID envelope has ceased to decay and starts to grow again, then this condition is associated with $u(t)$ being at a minimum, i.e. $\dot{u}(t)=0$ and $\ddot{u}(t)<0$, with $\dot{z}(t)<0$. The condition $\dot{u}(t)=0$ in Eqs. 2 yields $z=-\frac{\gamma_{2}}{\lambda}$. This means that the threshold condition at each burst is also associated to a stationary value of the magnetization $z$ component, $z^{s t}$. As will be detailed below, it indeed corresponds to a fixed point of the system.

- A maser burst can only occur if the characteristic time of radiation damping $\tau_{\mathrm{RD}}$ is such that $\tau_{\mathrm{RD}}^{-1}>\gamma_{2}$, that is, $\frac{\mu_{0} \gamma_{H} \eta Q}{2} \sqrt{z^{2}+u}>\gamma_{2}$.

- During a maser pulse, the non-linearity of Eqs. 2, which is associated to the growth of the transverse magnetization, becomes significant when the terms $\lambda u(t)$ and $\gamma_{n, e e} z(t)$ are of comparable magnitudes. In contrast, when $\lambda u(t) \ll \gamma_{n, e e} z(t)$, i.e. in the time interval between the end of a maser pulse and the onset of the next, one has $\dot{z}(t) \approx-\gamma_{n, e e}\left(z(t)-M_{z}^{s t}\right)$. This linear regime is associated to the exponential return of the $z$ component of the magnetization towards its (negatively) "hyperpolarized" value between pulses. This return to the "hyperpolarized" state is faster with increasing values of $\gamma_{n, e e}$, leading to bursts that are closer in time.

As stated above, the evolution at long times of the \{magnetization + inverse spin temperature\} system requires the determination of the fixed points (solutions of $\frac{d}{d t}=0$ ). However, these fixed points could only be found under the simplifying assumption of a transverse magnetization that relaxes to zero rather than to a noise value. The 
differential system Eq. 2 thus admits two solutions $F=\left(u^{s t}, z^{s t}, w^{s t}, \beta_{d}^{s t}\right)$ :

$$
\begin{aligned}
& F 1: \quad\left(0, \frac{w_{\mathrm{L}}^{0}\left(\gamma_{e e, \mathrm{~L}}+\Omega\right)}{\gamma_{e e, \mathrm{~L}}+\delta}, w^{s t}=z^{s t}, \beta_{d}^{s t}=\frac{1}{B_{h}} w^{s t}\right) \\
& F 2: \quad\left(-\frac{\gamma_{n, e e}\left[\frac{\gamma_{2}\left(\gamma_{e e, \mathrm{~L}}+\delta\right)+\lambda w_{\mathrm{L}}^{0}\left(\gamma_{e e, \mathrm{~L}}+\Omega\right)}{\lambda^{2}}\right],-\frac{\gamma_{2}}{\lambda}, \ldots}{\gamma_{e e, n}+\gamma_{e e, \mathrm{~L}}+\delta}\right] \\
&\left.\frac{\lambda w_{\mathrm{L}}^{0}\left(\gamma_{e e, \mathrm{~L}}+\Omega\right)-\gamma_{e e, n} \gamma_{2}}{\lambda\left(\gamma_{e e, n}+\gamma_{e e, \mathrm{~L}}+\delta\right)}, \frac{1}{B_{h}} w^{s t}\right)
\end{aligned}
$$

If we further restrict the study to the special case that corresponds to experiments where the microwave irradiation is switched off prior to FID detection, one has $\delta=$ $\Omega=0$ and $F 1=\left(0, w_{\mathrm{L}}^{0}, w_{\mathrm{L}}^{0}, w_{\mathrm{L}}^{0}\right)$, which just represents the thermal equilibrium. Alternatively, for $F 2$, the squared transverse magnetization $u(t)$ admits the value $u^{s t}<0$, which, from the definition of $u(t)$, is obviously impossible. Therefore, among both solutions, only $F 1$ is acceptable when the system is not under microwave irradiation. This means that at longer times the magnetization is necessarily brought back to its equilibrium position.

There are, however, two limiting cases that lead to $u^{s t} \rightarrow 0$, for which $F 2$ becomes acceptable. A scenario would be that the relaxation rate of the electron dipole reservoir is vanishingly small, $\gamma_{e e, \mathrm{~L}} \rightarrow 0$. This is clearly unrealistic, as $\tau_{e e, \mathrm{~L}}=\gamma_{e e, \mathrm{~L}}^{-1}$ is typically on the order of $\sim 10-100 \mathrm{~ms}$, and this assumption must therefore be discarded.

But the more interesting and plausible case is the situation where the rate of temperature transfer from the electron dipole reservoir to the ${ }^{1} \mathrm{H}$ nuclear spin reservoir is much faster than the equilibration rate between the dipolar electron spin reservoir and the lattice: $\gamma_{e e, n} \gg \gamma_{e e, \mathrm{~L}}$. Such a condition is met whenever the heat capacity $c_{H}$ of the ${ }^{1} \mathrm{H}$ nuclear spin reservoir is much larger than that of the electron dipolar (nonZeeman) reservoir, $c_{\mathrm{E}}$. The temperature flux from the dipole reservoir to the lattice is therefore quenched for the benefit of the ${ }^{1} \mathrm{H}$ nuclear spins. This implies that the ratio $\gamma_{n, e e} / \gamma_{e e, n}=c_{\mathrm{E}} / c_{\mathrm{H}}$ is vanishingly small, so that one has $\gamma_{e e, n} \gg \gamma_{e e, \mathrm{~L}}$. Moreover, the maser threshold condition implies (see below) $\gamma_{2} \sim \lambda w^{\text {thres }}(t)$, so that $\gamma_{2} \gg \lambda w_{\mathrm{L}}^{0}$. These assumptions therefore lead to $F 2:\left(0,-\frac{\gamma_{2}}{\lambda},-\frac{\gamma_{2}}{\lambda},-\frac{\gamma_{2}}{\lambda B_{h}}\right)$, a non-Boltzmann stationary solution. 
The long-time evolution of the \{magnetization + spin temperature\} system is investigated from the linearized Eqs. 2 in the vicinity of the fixed points. In particular, the stability of the latter depends on the signs of the real parts of the eigenvalues of the linearized system (for a complete reference, see for instance ${ }^{29}$ ). Using the change of variables: $u(t)=U(t)+u^{s t}, z(t)=Z(t)+z^{s t}, w(t)=W(t)+w^{s t}, \beta_{d}(t)=R(t)+\beta_{d}^{s t}$, Eq. 2 becomes:

$$
\left(\begin{array}{c}
\dot{U} \\
\dot{Z} \\
\dot{W} \\
\dot{R}
\end{array}\right)=\left(\begin{array}{cccc}
-2\left(\lambda z^{s t}+\gamma_{2}\right) & -2 \lambda u^{s t} & 0 & 0 \\
\lambda & -\gamma_{n, e e} & \gamma_{n, e e} & 0 \\
0 & b & a & d \\
0 & 0 & \gamma_{d, e e} / B_{h} & -\gamma_{d, e e}
\end{array}\right)\left(\begin{array}{c}
U \\
Z \\
W \\
R
\end{array}\right)+\left(\begin{array}{c}
-2 \lambda Z U \\
0 \\
0 \\
0
\end{array}\right)
$$

Thus, in the vicinity of $F 2$, the eigenmodes of the linear part of Eq. 4 are given by the roots of the determinant (since $\lambda z^{s t}+\gamma_{2}=0$ ):

$$
\left|\begin{array}{ccc}
x+\gamma_{n, e e} & -\gamma_{n, e e} & 0 \\
-\gamma_{e e, n} & x+\gamma_{e e, n}+\gamma_{e e, d} & -B_{h} \gamma_{e e, d} \\
0 & -\gamma_{d, e e} / B_{h} & x+\gamma_{d, e e}
\end{array}\right|
$$

,or: $x=0$ and $x_{ \pm}=\frac{-\left(\gamma_{n, e e}+\gamma_{e e, n}+\gamma_{e e, d}+\gamma_{d, e e}\right) \pm \sqrt{\Delta}}{2}$, where $\Delta=\left(\gamma_{n, e e}+\gamma_{e e, n}\right)^{2}-$ $2 \gamma_{e e, d}\left(\gamma_{n, e e}-\gamma_{e e, n}\right)-2 \gamma_{d, e e}\left(\gamma_{n, e e}+\gamma_{e e, n}\right)+\left(\gamma_{e e, d}+\gamma_{d, e e}\right)^{2}$. Moreover, since $\gamma_{n, e e}, \gamma_{e e, n}, \gamma_{e e, d}, \gamma_{d, e e} \geq$ 0 , one sees that $\Delta \leq\left(\gamma_{n, e e}+\gamma_{e e, n}+\gamma_{e e, d}+\gamma_{d, e e}\right)^{2}$. When $\Delta>0$, both eigenvalues of the linear part of Eq. 4 are negative, $x_{ \pm} \leq 0$, and $F 2$ is a stable asymptotic equilibrium. And if $\Delta<0$, both $x_{ \pm}$have a negative real part so that $F 2$ is also stable. It is noteworthy that, although the fixed points do not depend on the value of $\gamma_{e e, d}$ (or $\gamma_{d, e e}$ ), the latter partially determine the kinetics of the approach to equilibrium. For large heat capacities $c_{\mathrm{D}}$, comparable to $c_{\mathrm{H}}$, which is the case for large quantities of ${ }^{2} \mathrm{H}$ nuclei, both $\gamma_{e e, d}$ and $\gamma_{d, e e}$ contribute significantly. Thus, in an idealized case where the ratio $c_{\mathrm{H}} / c_{\mathrm{E}}$ is assumed infinitely large and ${ }^{1} \mathrm{H} T_{1}$ relaxation is negligible, the magnetization does not return to thermal equilibrium, once trapped in the basin of attraction of $F 2$. This therefore suggests that in actual experiments an out-of-equilibrium magnetiza- 
tion can be maintained for a significant period of time, even without $\mu \mathrm{w}$ irradiation. Alternatively, in the presence of $\mu \mathrm{w}, F 2$ is an admissible solution (if $u_{s t} \geq 0$, see Eq. 3), depending on the irradiation offset and power of the microwave field. Due to the complexity of the BMP equations, the study of the magnetization dynamics requires numerical simulations. For the sake of illustration, parameters similar with the ones expected in our experiments were used. The magnetization dynamics are illustrated in Figure 3, where $u(t)$ and $M_{z}$ are depicted. These graphs show the succession of bursts at short times $(<0.1 \mathrm{~s})$ followed by a very slow decay. Simulations also show that $-\gamma_{2} / \lambda$ is not a fixed point. This is expected, as the condition $c_{H} / c_{e}=\infty$ cannot be exactly fulfilled. However, it is seen to be transiently the case, and only when the in-plane component becomes null does the longitudinal magnetization relax towards thermal equilibrium. During this succession of maser pulses, the $M_{z}$ component of the magnetization remains negative. This is consistent with the analysis of the magnetization dynamics in usual NMR experiments in the presence of radiation damping. For the case of RD in the absence of $T_{1}$ relaxation - where a single maser burst is present - analytical solutions of the magnetization dynamics ${ }^{30}$ show that the end point of the trajectory of the magnetization vector reaches the north pole of the Bloch sphere only when $T_{2}$ is infinite. However, the $z$-value of the final magnetization decreases as $T_{2}$ decreases. It can even become negative, depending on the relative values of $T_{2}$ and $\tau_{\mathrm{RD}}$, when defocusing is too fast to maintain an efficient RD throughout. This behavior is confirmed using the analytical expressions of the longitudinal magnetization derived by Bloom and the parameters relevant to the present work (not shown). Finally, the presence of a noise term, discarded in the qualitative analysis, was introduced in the simulations. In this case, even the analytical determination of the stationary solutions is beyond reach, but simulations clearly demonstrate the significant effect of the noise voltage, therefore transverse magnetization fluctuations. A comparison between simulations performed in the presence and in the absence of this noise term is shown in Figure 4. This term has two major effects on the dynamics: i) during the early stage of the FID, the maser bursts are of lesser amplitude and number; and ii) the transverse 
magnetization reaches its stationary value significantly more slowly than in the absence of the noise term. This may be interpreted as the effect of the circuit noise that maintains an in-plane magnetization and keeps on driving radiation damping up to the point where $\sqrt{z^{2}+u}$ becomes practically zero.

The experiments such as those presented in Fig. 2 suggest the existence of a thermal contact between the ${ }^{1} \mathrm{H}$ and the electron spin dipolar reservoirs, as implied by the BMP model. Indeed, the observed dynamics require, in addition to an efficient coupling of the magnetization to the probe where magnetic energy is dissipated, some additional mechanism that drives the magnetization towards the negative direction of the $z$ axis. Obviously, this mechanism cannot be based on nuclear relaxation, since both the radiation damping feedback field and longitudinal relaxation tend to bring $\mathbf{M}$ back to $+z$. Thus, the existence of a process that drives $M_{z}$ to the $-z$ direction is necessary, i.e. a repolarization process towards negative values. Importantly, this repolarization occurs even in the absence of the $\mu \mathrm{w}$ field (see Fig. 2), which therefore demonstrates that polarization is provided to ${ }^{1} \mathrm{H}$ spins by the ${ }^{2} \mathrm{H}$ reservoir through the electron dipolar reservoir.

The multiple resurgence of the NMR signal attests for successive rebuilding of a radiation damping field. The simulations presented here show that, because of the remagnetization caused either by microwave irradiation or through the ${ }^{2} \mathrm{H}$ polarization reservoir (growing of the $M_{z}$ in magnitude towards a negative value), even a small residual in-plane component of the magnetization may be sufficient to make the coherent feedback field strong enough to trigger the next maser pulse. This process is reminiscent of the phenomenon described in ref., ${ }^{4}$ where $\pi$-pulses were used to generate "multiple echoes". In that case, the pulses served as a way to put the in-plane magnetization in coherence, thereby creating a large enough RD field at the time of in-plane refocusing thanks to the remaining negative $M_{z}$ to trigger a new maser pulse. However, in this context, the term "echo" may be somewhat misleading, because the signal observed is not simply the refocusing of in-plane magnetization. The refocusing of the in-plane magnetization achieved by the $\pi$-pulse creates a coherent RD field 
that triggers an actual maser pulse, provided that the remaining $M_{z}$ component is large enough in magnitude and negative. In our case, the magnitude of the magnetization vector is so large that the residual in-plane magnetization achieves the same effect. In this perspective, the observations definitely represent self-sustained, self-triggered series of maser pulses.

Fitting the experimental FIDs to the complete BMP model is rather challenging, as, clearly, the problem at hand is largely under-determined when only the measurements of the FIDs are available. Thus, the maser bursts at short times, typical of the nonlinear behaviour of the ${ }^{1} \mathrm{H}$ magnetization, are determined by the time constant $\tau_{\mathrm{RD}}^{-1}$, which results from the product of $m_{z}^{s t}, \eta Q$ and the magnetization (itself the product of the number of ${ }^{1} \mathrm{H}$ spins by the polarization). Moreover, the ${ }^{1} \mathrm{H}$ pulses may likely be miscalibrated in the presence of such large radiation damping feedback field. For these reasons, one does not expect to access the value of the magnetization from the NMR signal in these experiments. On a more fundamental level, the high-temperature approximation implied by the Provotorov equations may be violated in our experimental conditions. This is primarily relevant when microwave irradiation is active during the observation of the FID. When the $\mu \mathrm{w}$ field is turned off, the electron spin dipole reservoir is decoupled from the Zeeman electron spin reservoir, and so is the nuclear spin Zeeman reservoir, so that the high temperature approximation is verified and the description in terms of flows of inverse spin temperatures remains possible. However, during irradiation, such treatment may not be the valid, although for strong enough $\mu \mathrm{w}$, the electron Zeeman reservoir is warmed up during irradiation, which might still justify the high-temperature regime. Detailed discussions can be found in Ref. ${ }^{16,19}$ In view of these various difficulties, we introduced a simplified model in which it is assume that ${ }^{1} \mathrm{H}$ magnetization relaxes towards a time-dependent value that itself tends to $z^{\text {st }}$ mono-exponentially, with the rate $\gamma_{\text {depol }}$ :

$$
\begin{aligned}
\frac{d}{d t} M_{z}(t) & =\omega_{1} M_{y}+\gamma_{\mathrm{H}} G\left(M_{x}^{2}+M_{y}^{2}\right)-\gamma_{n, e e}\left(M_{z}-M_{z}^{a}(t)\right) \\
\frac{d}{d t} M_{z}^{a}(t) & =\gamma_{\text {depol }}\left(M_{z}^{a}(t)-M_{z}^{s t}\right)
\end{aligned}
$$


This can be justified, as discussed in the previous sections, when repolarization of the ${ }^{1} \mathrm{H}$ spins towards its initial hyperpolarized state is achieved through microwave irradiation, or, in the absence of the latter, when $\gamma_{e e, n}, \gamma_{e e, d} \gg \gamma_{e e, \mathrm{~L}}$. In this case, $\beta_{e e}$ undergoes fast equilibrium with the nuclear Zeeman reservoirs. With this model, the fitted parameters are the initial flip angle, $\gamma_{n, e e}, \eta Q$, the initial common spin temperature $T_{\text {spin }}$, the number of ${ }^{1} \mathrm{H}$ spins in the sample, and $M_{z}^{s t}$. A qualitative analysis of these equations is shown in the Supplementary Information. An illustrative experiment is shown in Fig. 5, where the NMR signal was recorded in the presence of the microwave. Fitting Eqs. 6 to the data yielded in this case $\gamma_{n, e e} \approx 10.1 \mathrm{~s}^{-1}$ and $\gamma_{\text {depol }} \approx 4.08 \mathrm{~s}^{-1}$. The RD time constant $\tau_{\mathrm{RD}} \approx 1 / \gamma_{2}$, which corresponds to the maser triggering condition, was also determined by fitting. This unusually short value of the radiation damping time for ${ }^{1} \mathrm{H}$ in a "water sample" is nevertheless explained by the huge $(\sim 4$ orders of magnitude) polarization gain afforded by DNP with respect to ambient temperature experiments.

Again, due to the relations between the parameters, only if $N_{\text {spin }}$ and $P^{s t}$ are known can the "true" value of $M_{z}^{s t}=-\gamma_{2} / \lambda$ be determined, which is why the quantities in Fig. 5 are plotted in arbitrary units. Moreover, fitting of this experimental FID yielded $M_{z}^{s t} \approx-\gamma_{2} / \lambda$, which corresponds to the fixed point $F 2$ of Eqs 2, the expected value in the absence of the noise term. This shows that the latter has little influence in this case. The present work describes the magnetization dynamics of a complex system of dipolar coupled nuclear ${ }^{1} \mathrm{H}$ spins using a simplistic Bloch vector description, neglecting the presence of nuclear dipolar interactions and their effects on the magnetization dynamics. These lead to the generation of higher ${ }^{1} \mathrm{H}-{ }^{1} \mathrm{H}$ spin order and to line narrowing with increasing polarization $P$. The associated line narrowing can be assessed by the second moment $m_{2}$ of the line (an estimate of the line width for a Gaussian line shape) which, in the special case of a spherical sample with cubic lattice, is $m_{2}(P)=$ $m_{2}(0)\left(1-P^{2}\right),{ }^{16,31,32}$ where $m_{2}(0)$ is the second moment in the high temperature approximation. Using $P \approx 90 \%$, and $m_{2}(0) \approx 25 \mathrm{kHz}$, one expects $m_{2}(P)$ on the order of $\approx 5 \mathrm{kHz}$. This gross estimate suggests a longer FID, with a complete decay in a few 
hundreds of microseconds, much shorter than the width of the observed signal bursts ( $\sim$ milliseconds). Such a contribution of higher spin orders may likely affect the details of the FIDs and add complexity to the problem.

In the experiments presented here, a crude estimate shows that the associated increased FID duration remains orders of magnitude shorter the duration of the signal bursts so that these effects can be neglected in our qualiative analysis of the nonlinear magnetization behaviour.. However, one can not discard the effects of nuclear spin dipolar couplings for a thoroughly quantitative analysis of these sustained maser pulses, and deserve a study of their own.

In Ref. ${ }^{14}$ the DNP process in a ruby crystal was analyzed in great detail, and estimates of the various parameters that appear in the Provotorov equations were provided through a series of complementary experiments. Maser oscillations were also observed, although the long-time regime was not analyzed in a quantitative manner and a simultaneous fit of the observed NMR signals on both short and long time scales - one of the results in the present work - was not performed. Here, an NMR maser was achieved in an amorphous sample of DNP-hyperpolarized nuclei, and the resulting NMR signal could be analyzed based on a simplified BMP model. In addition, the observation of sustained maser pulses even in the absence of $\mu \mathrm{w}$ irradiation and the analyses reported in this work provide further evidence that the re-magnetization of

${ }^{1} \mathrm{H}$ spins is provided by the ${ }^{2} \mathrm{H}$ reservoir present in the sample. Thus a single FID contains information relative to processes of different natures, namely RD and DNP. Additionally, RD may represent a potential tool to gain insight into the DNP mechanism by providing a way to access characteristic parameters of the DNP process itself. The presented analysis, although currently restricted to thermal mixing-type DNP, may generalize to other DNP mechanisms, such as the solid and the cross effect.

\section{Conflicts of interest}

There are no conflicts of interest to declare. 


\section{Acknowledgements}

E.W. and D.K. acknowledge funding from the European Research Council ("Dilute para-water", grant agreement No 339754). Bruker BioSpin is greatfully acknowledged for providing the D-DNP equipment.

\section{References}

(1)Bloembergen, N.; Pound, R. V. Physical Review 1954, 95, 8-12.

(2)Vlassenbroek, A.; Jeener, J.; Broakaert, P. Journal of Chemical Physics 1995, 103, 58865897.

(3)Dicke, R. H. Physical Review 1954, 93, 99-110.

(4)Hahn, E.; Augustine, M. J. Chem. Phys. 1997, 107, 3324.

(5)McCoy, M.; W.S.Warren, Journal of Chemical Physics 1990, 93, 858.

(6)Abergel, D.; Delsuc, M.-A.; Lallemand, J.-Y. Journal of Chemical Physics 1992, 96, 1657.

(7)Abergel, D.; Lallemand, J.-Y. J. Magn. Reson. A 1994, 110, 45.

(8)Chupp, T. E.; Hoare, R. J.; Walsworth, R. L.; Wu, B. Phys. Rev. Lett. 1994, 2363-2366.

(9)Marion, D. J.-Y.; Huber, G.; Berthault, P.; Desvaux, H. ChemPhysChem 2008, 9, 13951401.

(10)Abergel, D.; Louis-Joseph, A.; Lallemand, J.-Y. Journal of Chemical Physics 2002, 116, 7073-7080.

(11)Abergel, D.; Louis-Joseph, A. Journal Magnetic Resonance 2008, 196, 115-118.

(12)Kiselev, Y. F.; Prudkoglyad, A. F.; Shumovskii, A. S.; Yukalov, V. I. Sov.Phys.JETP 1988, 67, 413-415. 
(13)Reithertz, L. A.; Dutz, H.; Goertz, S.; Krämer, D.; Meyer, W.; Reitherz, G.; Thiel, W.; .Thomas, A. Nuclear Instruments and Methods in Physics Research 1994, A 340, 278282.

(14)Bosiger, P.; Brun, E.; Meier, D. Physical Review A 1978, 18, 671-684.

(15)Bosiger, P.; Brun, E.; Meier, D. Phys. Rev. Lett. 1977, 38, 602-605.

(16)Abragam, A.; Goldman, M. Reports on Progress in Physics 1978, 41, 395-467.

(17)Atsarkin, V.; Rodak, M. Soviet Physics Uspekhi 1972, 15, 251-374.

(18)Atsarkin, V. Soviet Physics Uspekhi 1978, 21, 725-745.

(19)Wenckebach, T. Essentials of Dynamic Nuclear Polarization; Spindrift Publications, 2016.

(20)Ardenkjaer-Larsen, J. H.; Fridlund, B.; Gram, A.; Hansson, G.; Hansson, L.; Lerche, M. H.; Servin, R.; Thaning, M.; Golman, K. Proc. Natl. Acad. Sci. U. S. A. 2003, 100, 10158-10163.

(21)Becerra, L. R.; Gerfen, G. J.; Temkin, R. J.; Singel, D. J.; Griffin, R. G. Phys. Rev. Lett. 1993, $71,3561-3564$.

(22)Wenckebach, W. Journal of Magnetic Resonance 2019, 299, 124-134.

(23)Wenckebach, W. Journal of Magnetic Resonance 2019, 299, 151-167.

(24)Provotorov, B. N. Soviet Physics JETP 1962, 14, 1126-1131.

(25)Provotorov, B. N. Soviet Physics JETP 1962, 15, 611-614.

(26)Goldman, M. Spin Temperature and Nuclear Magnetic Resonance in Solids; Clarendon, Oxford, 1970.

(27)Bloom, S. Journal of Applied Physics 1957, 28, 800-805.

(28)Vlassenbroek, A.; Jeener, A.; Broekaert, P. Journal of Chemical Physics 1995, 103, 58865897. 
(29)Guckenheimer, J.; Holmes, P. Nonlinear Oscillations, Dynamical Systems, and Bifurcations of Vector Fields; Applied Mathematical Sciences 42; Springer, 1983.

(30)Bloom, S. Journal of Applied Physics 1957, 28, 800.

(31)Abragam, A.; Goldman, M. Journal of magnetic resonance 1973, 10, 322-346.

(32)Walls, J. D.; Phoa, F. K. H.; Lin, Y.-Y. Physical Review B 2004, 70, 174410. 


\section{Figures}

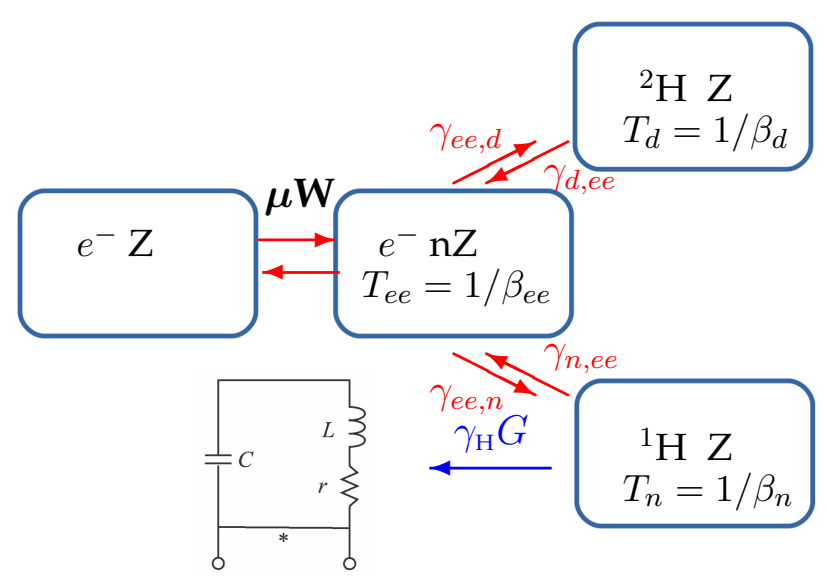

Figure 1: The TM-RD setup for a sample containing ${ }^{1} \mathrm{H}$ and ${ }^{2} \mathrm{H}$ nuclear spins. The letters $\mathrm{Z}$ and $\mathrm{nZ}$ respectively refer to Zeeman and non-Zeeman reservoirs, according to the theory of thermal mixing. Both nuclear Zeeman and the electronic dipolar reservoirs are coupled. Microwave irradiation of the EPR line couples the electron spin Zeeman and dipolar reservoirs. ${ }^{1} \mathrm{H}$ magnetization is lost to the NMR probe, which acts as a sink for the former. 


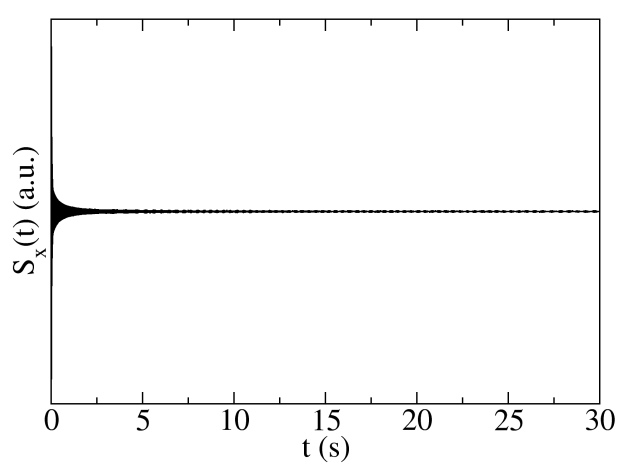

(a)

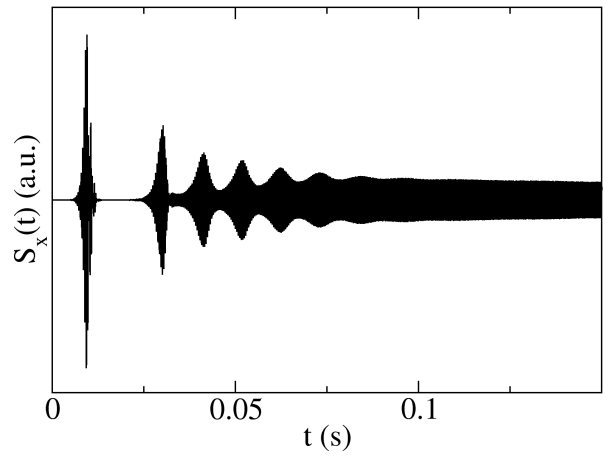

(c)

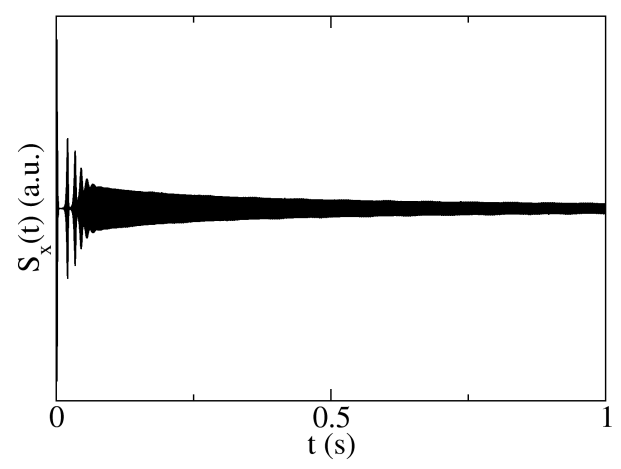

(b)

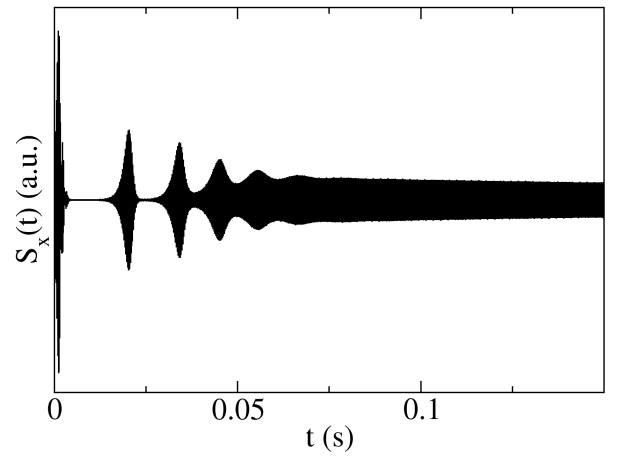

(d)

Figure 2: Typical FIDs following an initial polarisation period of $\sim 5 \mathrm{~min}$. In 2(a) and 2(c), the signal was triggered by noise, as no rf pulse was applied. In 2(b) and 2(d), a small flip angle pulse was applied $\left(0.1^{\circ}\right)$. After sustained maser pulses have decayed the signal remains nonzero for several seconds, indicating the absence of return to equilibrium of the ${ }^{1} \mathrm{H}$ magnetization. 


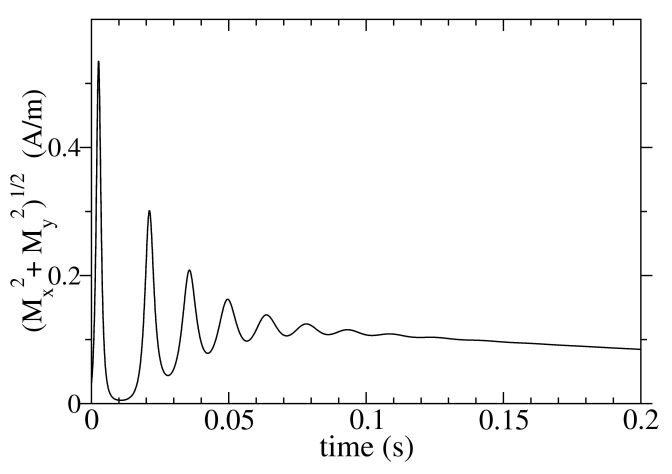

(a)

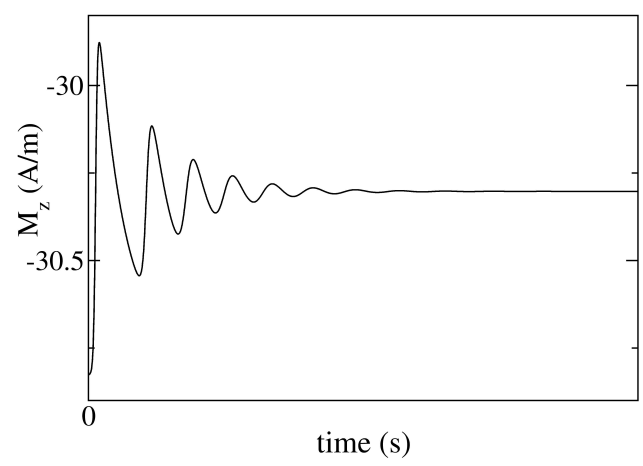

(c)

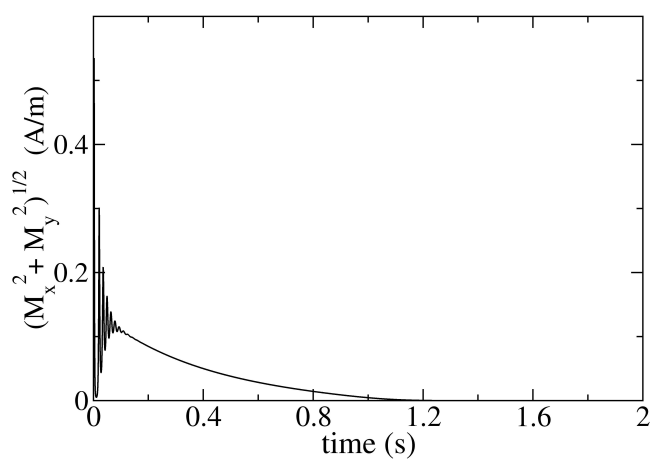

(b)

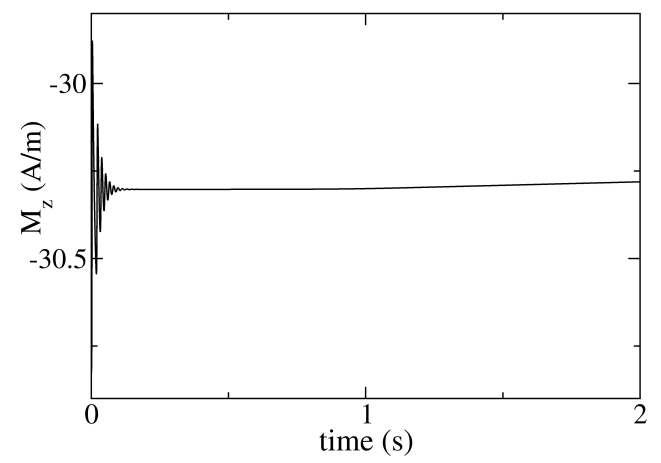

(d)

Figure 3: Simulations of the BMP equations in the absence of $\mu \mathrm{w}$ irradiation and transverse thermal noise component. In (a) and (b), and (c) and (d), the magnitude of the transverse component and the longitudinal component of the magnetization are depicted, respectively. Spin densities were $N_{e} \approx 30 \cdot 10^{24} \mathrm{~m}^{-3}, N_{1_{\mathrm{H}}} \approx 6.6 \cdot 10^{27} \mathrm{~m}^{-3}$, and $N_{2_{\mathrm{H}}} \approx 54.5 \cdot 10^{27} \mathrm{~m}^{-3}$. Heat capacities were: $c_{\mathrm{X}}=N_{\mathrm{X}} I(I+1)\left(\frac{\omega_{\mathrm{X}}}{2 \pi}\right)^{2}$, ${ }^{17}$ where $\mathrm{X}={ }^{1} \mathrm{H},{ }^{2} \mathrm{H}, I=1 / 2,1$ and $\omega_{\mathrm{X}} / 2 \pi$ is the resonance frequency of spin $\mathrm{X}$. Moreover, $\gamma_{n, e e}=200 \mathrm{~s}^{-1}$ and $\gamma_{d, e e}=10 \mathrm{~s}^{-1}$ were used and the read pulse flip angle $\theta=10^{-3}$. The transverse relaxation rate was set to $\gamma_{2}=2 \pi \times 25 \mathrm{kHz}$. The values of $\eta$ and $Q$ were chosen so that $\tau_{\mathrm{RD}} \approx 1 / \gamma_{2}(\eta Q=15.4)$, a necessary condition for multiple maser oscillations. The EPR linewidth was set to $\omega_{s s}=1.25 \cdot 10^{9} \mathrm{~s}^{-1}$. The initial spin temperature, when microwave irradiation is turned off was $T_{\text {spin }}=-20 \mathrm{mK}$, and the lattice relaxation rate of the electron spin dipolar reservoir was set to $\gamma_{e e, \mathrm{~L}}=6 \mathrm{~s}^{-1}$. 


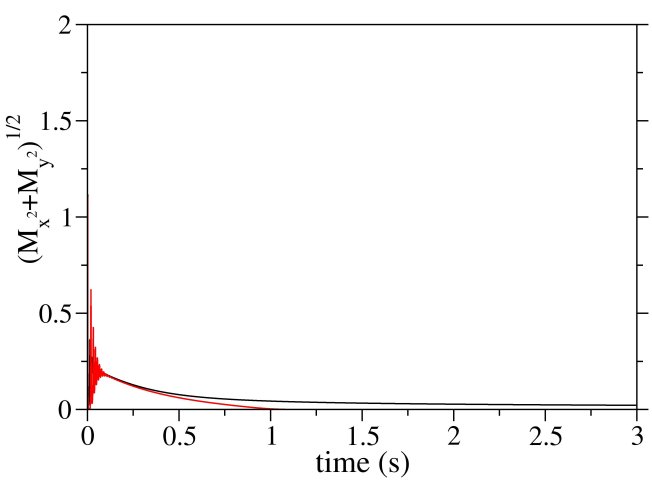

(a)

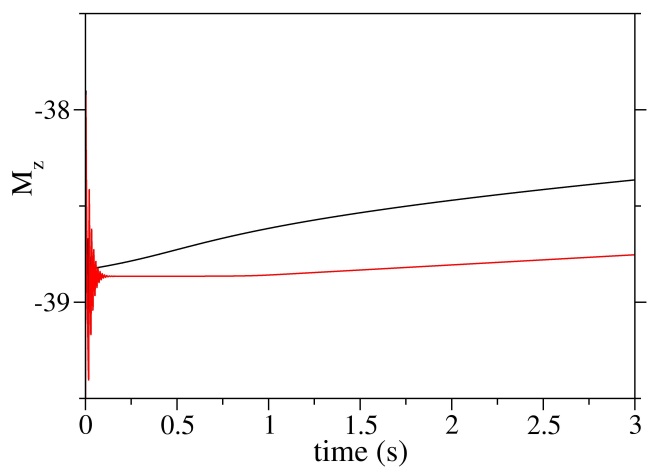

(c)

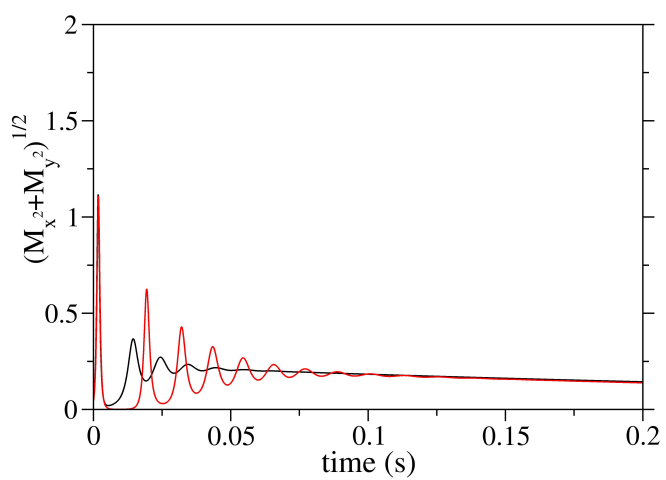

(b)

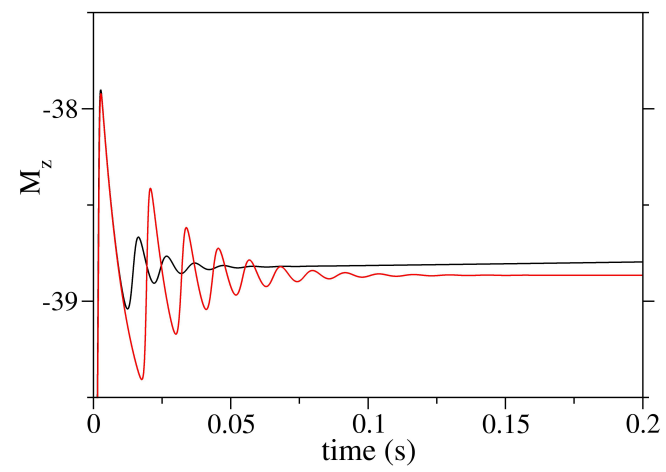

(d)

Figure 4: Simulations of the BMP equations performed in the absence (red) and in the presence of a thermal noise level of the transverse magnetization. Computations were performed with the parameters $\theta=10^{-3}, \eta Q=12$ and $\gamma_{n, e e}=100 \mathrm{~s}^{-1}$. The EPR linewidth was set to $\omega_{s s}=$ $1.8 \cdot 10^{9} \mathrm{~s}^{-1}$. The spin temperature in the initial state, when microwave irradiation is turned off is $T_{\text {spin }}=-15 \mathrm{mK}$. The transverse relaxation rate was $\gamma_{2}=2 \pi \times 25 \mathrm{kHz}$, the lattice relaxation rate of the electron spin dipolar reservoir was set to $\gamma_{e e, \mathrm{~L}}=6 \mathrm{~s}^{-1}$, and $\gamma_{d, e e}=10^{3} \mathrm{~s}^{-1}$. 

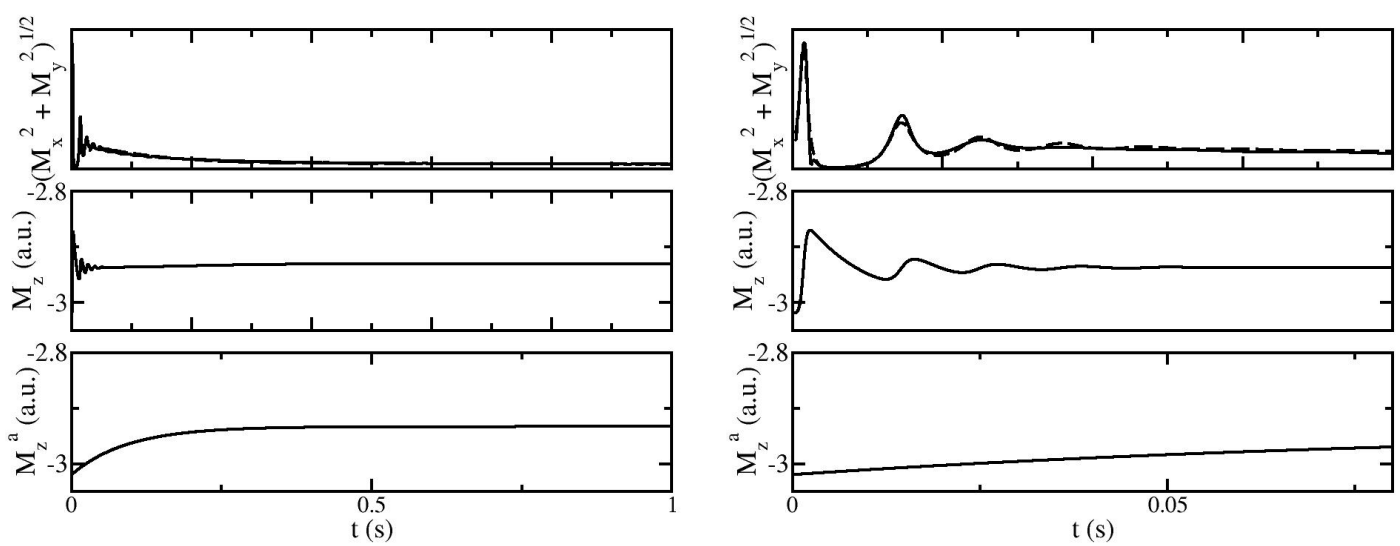

Figure 5: Experimental FID (solid line) obtained in the presence of microwave irradiation. Fitting (dashed line for the transverse magnetization) was obtained using the simplified model of Eq. 6. The full length FID is presented in the left panel, and a focus on the early times, where multiple MASER pulses occur, is shown on the right. As explained in the text, due to the relations between the parameters, the "true" value of $M_{z}^{s t}=-\gamma_{2} / \lambda$ cannot be determined in these experiments, and the quantities in Fig. 5 are plotted in arbitrary units. However, during the evolution, $M_{z}$ remains negative. 